\title{
Treatment of patients with advanced diabetes type 2 and hypertension without the use of conventional treatment is possible: white matter alterations and treatment of diabetes and hypertnsion as a new medical hypothesis
}

\begin{abstract}
Diabetes has been described for 3500 years. Therapeutic options vary but they involve giving insulin or lowering glucose such as metformin. The objective of this study was to report how diabetes type 2 and hypertension can be treated successfully without using the diabetes conventional treatments. A 50 years male with high glucose level $(330 \mathrm{mg} /$ dl), HBA1c (9.6\%). Blood profile was altered. Hypertension was elevated $(145 / 100) \mathrm{mm}$ hg. The patient expressed his tension and desire not to commit conventional diabetic and hypertension treatment and asked for other therapeutic alternatives. Based on our experience, we recommended him to use two spoon of ground Urtica pilulifera (U. $p$ )/ day, and to use two capsules of Ammi visnaga (A.V) following each meal. Further, the patient followed the dietary restrictions by replacing white bread with a spoon and followed some exercise programs by walking for 30 minutes a day. The results of this program were shown as continuous lowering blood glucose to the level of $117 \mathrm{mg} / \mathrm{dl}$, and blood pressure was $123 / 79$ after 5 days. Taken together, both diabetes type 2 and hypertension can be successfully treated naturally.
\end{abstract}

Keywords: diabetes type 2, hypertension, Ammi visnaga, Urtica pilulifera, glucose
Volume II Issue I - 202 I

\author{
Ahed J Alkhatib ${ }^{1,2}$ \\ 'Department of Legal Medicine,Toxicology and Forensic \\ Medicine, Jordan University of Science \& Technology, Jordan \\ ${ }^{2}$ Department of medicine and critical care, department of \\ philosophy, Academician secretary of department of Sociology, \\ Jordan
}

Correspondence: Ahed J Alkhatib, Department of Legal Medicine, Toxicology and Forensic Medicine, Jordan University Of Science \& Technology, Jordan, Tel 00962795905 I45, Emailajalkhati@just.edu.jo,drahedalkati@yahoo.com

Received: February 15, 2021 | Published: February 26, 2021

\section{Introduction}

Diabetes type 2 has been known for 3500 years. ${ }^{1}$ Its prevalence has increased over the time, in 2018 there were more than 500 million prevalent cases of type 2 diabetes worldwide. Prevalence will increase in all countries. ${ }^{2}$ Research findings showed the existence of a significant relationship between diabetes and obesity, insulin resistance and disturbances in the function of $\beta$-cells in the pancreas. ${ }^{3}$ Diabetic influences include lowering rates of insulin metabolism such as glucose, lipids, and proteins. ${ }^{4}$ The treatment options for diabetes type 2 vary and include diet control, physical activity, and blood glucose lowering agents such as metformin. ${ }^{5}$

Our previous studies showed thar several considerations regarding the etiology of diabetes and its potential treatments. We have previously demonstrated that alterations in white matter of diabetic rats included increased expression of inducible nitric oxide synthase (iNOS) and decreased expression of heat shock protein70 (HSP70). ${ }^{6}$

Urtica pilulefera (Roman nettle plant) belongs to the (Urticaceae) family, and it is distributed in the temperate region of the world, and contains various phytochemicals such as terpeniods, flavonoids, vitamins, etc. These types exercise diverse and effective pharmacological procedures for the management of various diseases such as cancer, diabetes, arthritis, etc. ${ }^{7}$ Urtica pilulefera has phenolic compounds such as quinic acid, caffeic acid, caffeoylquinic acid, decafoyl malic derivatives, apigenin glucoside (flavone) derivatives, and genistein glucoside (isoflavones). ${ }^{8}$

Ammi visnaga L. (Visnaga daucoides Gaertn., Family Apiaceae), also known as Khella Baldi or toothpick herb, is an annual or biennial herb indigenous to the Mediterranean region of North Africa, Asia and Europe. The plant is known to have been used in traditional medicine for a long time. Currently, it is used in modern medicine to treat many conditions such as renal colic and coronary heart failure, and is used as an antioxidant, antifungal and antibacterial, with a larvicidal effect on mosquito larvae. Peer-reviewed studies show that these pharmacological activities are due to their valuable chemical components that mainly include essential oil, polyphenolic compounds that include flavonoids, as well as $\gamma$-pyrones, represented mainly by khellin and visnagin. Its essential oil is reported to have antiviral, antibacterial, and larvicidal effects, while its flavonoid content is responsible for its antioxidant activity. Its $\gamma$-pyrone content has a powerful effect to facilitate the passage of kidney stones and alleviate kidney colic, as well as having a relaxing effect on smooth muscle including that of the coronary arteries. ${ }^{9}$

Tadalafil is a member of the phosphodiesterase- 5 inhibitor and is widely used to treat erectile dysfunction due to its long-acting properties. ${ }^{10}$ Tadalafil use has been associated with blood and tissue antioxidant activities. ${ }^{11}$ Some benefits were reported from daily use of Tadalafil $5 \mathrm{mg}$, such as increased regional cerebral blood flow (CBF) and improvement of cognitive function among patients with erectile dysfunction (ED) and those with mild cognitive impairment. ${ }^{12}$ We also previously found that in a model of diabetic mice, downregulation of the heat shock protein in white matter. ${ }^{13}$ We have recently reported the possibility of treating prediabetes using Urtica pilulifera using rat as an animal model. ${ }^{14}$ ). We also showed the possibility of treating prediabetes on human subjects using tadalafil $5 \mathrm{mg} /$ day. 


\section{Study objective}

The main objective of this study was to report how diabetes type 2 and hypertension can be treated successfully without using the conventional treatments of diabetes and hypertension.

\section{Case presentation}

A man, 50 years age, suffering from elevated blood fasting sugar $(330 \mathrm{mg} / \mathrm{dl})$, HBA1c $(9.6 \%)$, and blood pressure (145/100 hg), and his lipid profile was disturbed. The patient was reluctant to use conventional treatment because he believed that starting conventional treatment meant continuing to take these treatments throughout his life. The patient was advised to follow the following protocol: two food spoons of ground powder of U. P, one spoon on the morning, and evening. Two capsules of A.V after each meal. Walking for 30 minutes daily. White bread was not allowed through this program. The readings of fasting glucose and blood pressure were daily taken in the morning.

\section{Results of the study}

The blood glucose levels began to decline immediately, and after 10 days glucose level was $117 \mathrm{mg} / \mathrm{dl}$, and the blood pressure reading was $123 / 78 \mathrm{~mm}$ hg (Figure 1). As shown in Table $1 \&$ Figure 2, the mean level of systolic pressure declined from 145 to $134 \mathrm{~mm} \mathrm{hg}$, whereas the mean of diastolic pressure declined from 100 to $85,5 \mathrm{~mm}$ hg. The mean level of glucose declined from $330 \mathrm{mg} / \mathrm{dl}$ to $130 \mathrm{mg} /$ dl. ${ }^{15}$

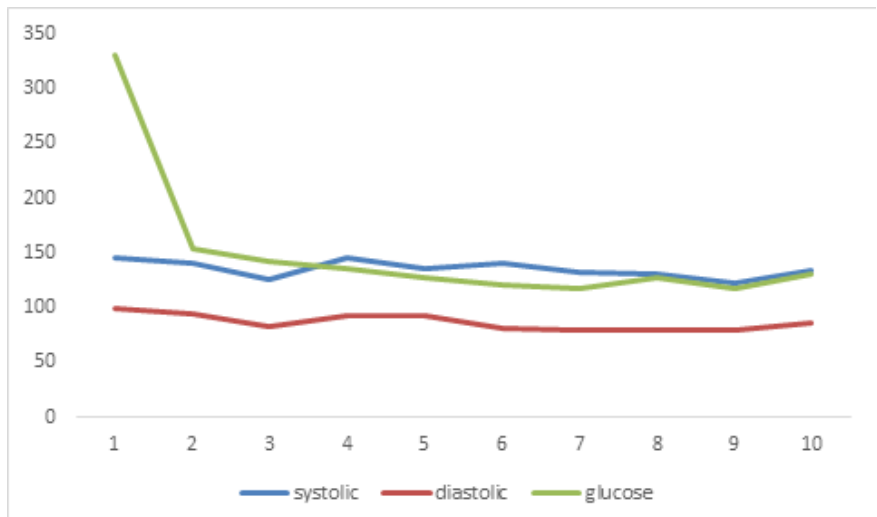

Figure I Readings of study variables through study period.

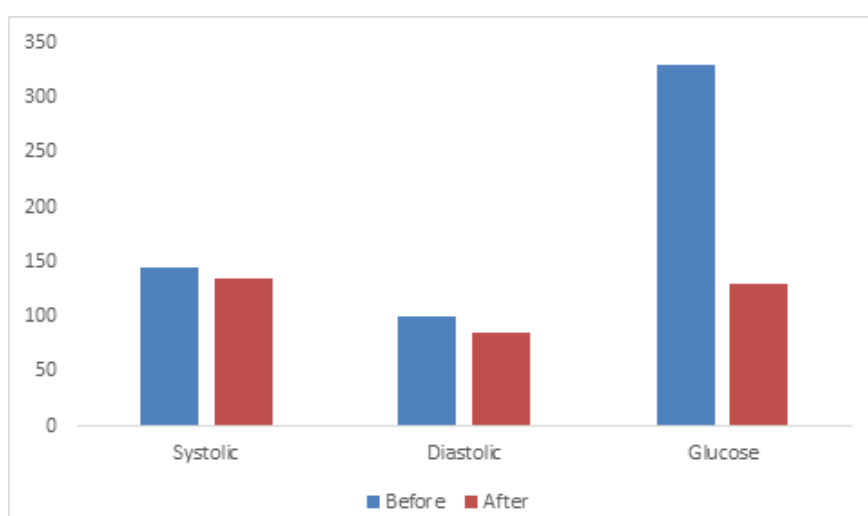

Figure $\mathbf{2}$ The mean levels of study variables before and after the study.
Table I The mean of readings before and after applying the nonpharmacological program

\begin{tabular}{llll}
\hline Variable & Systolic & Diastolic & Glucose \\
\hline Before & 145 & 100 & 330 \\
After & 134 & 85.5 & 130
\end{tabular}

\section{Discussion}

The results of the present study showed that hypertension and diabetes can be managed naturally without using medications. We based this strategy on our previous studies in which diabetes have impacts on white matter through down regulation of HSP70 and upregulation of iNOS. ${ }^{6}$ This implies that if we can reverse this pathway, it is possible to benefit the persons with diabetes. We have previously shown that prediabetes can be reversed through using $\mathrm{U}$. $\mathrm{P}^{14}$ using animal models. We also showed that it is possible to reverse the status of prediabetes among subjects using low dose of tadalafil. ${ }^{12}$ Taken together, we think that the use of herbs such as U. P, and A. V that have several therapeutic properties such as various antioxidants and anti-inflammatory properties can reverse diabetic status through the up-regulation of HSP70 and down-regulation of iNOS.

\section{Conclusion}

Diabetes type 2 and hypertension can be treated using nonpharmacological approaches based on our hypothesis indicating that reversing injuries of white matter on molecular level can participate in treating hypertension and diabetes.

\section{Acknowledgments}

None.

\section{Conflicts of interest}

Author declare that there is no conflict of interest.

\section{Funding}

None.

\section{References}

1. SP Lasker, CS McLachlan, L Wang, et al. Discovery, treatment and management of diabetes. Journal of Diabetology. 2010;1:1.

2. Amy Bradshaw Kaiser, Nicole Zhang, Wouter Van Der Pluijm. Global Prevalence of Type 2 Diabetes over the Next Ten Years (2018-2028). American Diabetes Association. 2018.

3. Wang Lianzi, Tao Li, Liu Jiaqing, et al. Association between glycosylated hemoglobin A1c and bone biochemical markers in type 2 diabetic postmenopausal women: a cross-sectional study. BMC Endocrine Disorders. 2019;19:31

4. Skovso Sos. Modeling type 2 diabetes in rats using high fat diet and streptozotocin. J Diabetes Invest. 2014;5:349-358.

5. https://www.who.int/news-room/fact-sheets/detail/diabetes

6. Ahed J Al-khatib A. Co-expression of iNOS and HSP70 in diabetes type 1 makes a rational hypothesis to explain the diabetic neuropathy. European Scientific Journal. 2013; 9(3):145-156.

7. Maha N Hamad, Noor S Jaafar, Ibrahim S Abbas, et al. Comparison of the phenolic contents between Urtica dioica and Urtica pilulifera cultivated in Iraq by HPLC and HPTLC. Journal of International Pharmaceutical Research. 2019;45:111-122. 
8. Mahmoud AH. Does Urtica Pilulifera have anti-schistosomiasis mansoni activity? International Journal of Chemical, Environmental \& Biological Sciences. 2013;1(3): 2320-4079.

9. Khalil N, Bishr M, Desouky S, et al. A Potential Medicinal Plant: A Review. Molecules. 2020;25(2):301.

10. Coward RM, Carson CC. Tadalafil in the treatment of erectile dysfunction. Ther Clin Risk Manag. 2008;4(6):1315-1330.

11. Jin Bong Choi, Kang Jun Cho, Joon Chul Kim, et al. The Effect of Daily Low Dose Tadalafil on Cerebral Perfusion and Cognition in Patients with Erectile Dysfunction and Mild Cognitive Impairment. Clinical Psychopharmacology and Neuroscience. 2019;17(3):432-437.

12. Ahed J Alkhatib. Prediabetes can be Reversed Using Low Dose Tadalafil: Non- Classical Treatment of Diabetes as A New Medical Hypothesis. Archives of Diabetes \& Obesity. 2020;2(5).
13. Ahed J Alkhatib. New Insights to Diabetes: Is Diabetes a Metabolic Disorder or a Neurological Disease? International Journal of Diabetes \& Metabolic Disorders. 2019;4(1):1-2.

14. Amawi KF, Alkhatib AJ. (Urtica Pilulifera in Treating Pre-diabetic Rat Model to Control the Blood Glucose, Lipids and Oxidative Stress. Medical archives (Sarajevo, Bosnia and Herzegovina). 2020;74(3):168171.

15. Kawther Faisal Amawi, Inas Saleh Al Mazari, Ali Alsarhan, et al. Diabetes upregulates the expression of HSP90 and downregulates HSP70 in the liver of diabetic rats. Comp Clin Pathol. 2019;28:473-478. 